\title{
Exploring the partonic phase at finite chemical potential within an extended off-shell transport approach
}

\author{
Elena Bratkovskaya $^{1,2}$ | Wolfgang Cassing ${ }^{3}$ | Pierre Moreau ${ }^{2}$ | Lucia Oliva ${ }^{2}$ \\ Olga Soloveva $^{2}$ | Taesoo Song ${ }^{1}$
}

${ }^{1}$ GSI, Helmholtzzentrum für

Schwerionenforschung $\mathrm{GmbH}$,

Darmstadt, Germany

${ }^{2}$ Institute for Theoretical Physics, University of Frankfurt, Frankfurt,

Germany

${ }^{3}$ Institute for Theoretical Physics,

University of Giessen, Giessen, Germany

\section{Correspondence}

Elena Bratkovskaya, GSI,

Helmholtzzentrum für

Schwerionenforschung $\mathrm{GmbH}$,

Darmstadt, Germany.

Email: e.bratkovskaya@gsi.de

\section{Funding information}

Deutsche Forschungsgemeinschaft (DFG, German Research Foundation), CRC-TR

211

\begin{abstract}
We extend the parton-hadron-string dynamics (PHSD) transport approach in the partonic sector by explicitly calculating the total and differential partonic scattering cross sections as a function of temperature $T$ and baryon chemical potential $\mu_{\mathrm{B}}$ on the basis of the effective propagators and couplings from the dynamical quasiparticle model (DQPM) that is matched to reproduce the equation of state of the partonic system above the deconfinement temperature $T_{\mathrm{c}}$ from lattice quantum chromodynamics (QCD). We calculate the collisional widths for the partonic degrees of freedom at finite $T$ and $\mu_{\mathrm{B}}$ in the time-like sector and conclude that the quasiparticle limit holds sufficiently well. Furthermore, the ratio of shear viscosity $\eta$ over entropy density $s$, that is, $\eta / s$, is evaluated using the collisional widths and compared to lattice QCD(1QCD) calculations for $\mu_{\mathrm{B}}=0$ as well. We find that the ratio $\eta / s$ does not differ very much from that calculated within the original DQPM on the basis of the Kubo formalism. Furthermore, there is only a very modest change of $\eta / s$ with the baryon chemical $\mu_{\mathrm{B}}$ as a function of the scaled temperature $T / T_{\mathrm{c}}\left(\mu_{\mathrm{B}}\right)$. This also holds for a variety of hadronic observables from central $\mathrm{A}+\mathrm{A}$ collisions in the energy range $5 \mathrm{GeV}$ $\leq \sqrt{s_{N N}} \leq 200 \mathrm{GeV}$ when implementing the differential cross sections into the PHSD approach. Accordingly, it will be difficult to extract finite $\mu_{\mathrm{B}}$ signals from the partonic dynamics based on "bulk" observables.
\end{abstract}

\section{K E Y W O R D S}

heavy ions, quark-gluon plasma, transport models

\section{1 | INTRODUCTION}

The phase transition from partonic degrees of freedom (quarks and gluons) to interacting hadrons is a central topic of modern high-energy physics. In order to

[Correction added on 27 October, after first online publication: Projekt Deal funding statement has been added.] understand the dynamics and relevant scales of this transition, laboratory experiments under controlled conditions are performed with relativistic nucleus-nucleus collisions. Hadronic spectra and relative hadron abundances from these experiments reflect important aspects of the dynamics in the hot and dense zone formed in the early phase of the reaction, and collective flows provide information on the transport properties of the medium 
generated on short time scales. As relativistic heavy-ion collisions start with impinging nuclei in their ground states, a proper nonequilibrium description of the entire dynamics through possibly different phases up to the final asymptotic hadronic states-eventually showing some degree of equilibration-is mandatory. To this aim, the parton-hadron-string dynamics (PHSD) transport approach (Bratkovskaya et al. 2011; Linnyk et al. 2013) has been formulated about a decade ago and was found to well describe observables from $\mathrm{p}+\mathrm{A}$ and $\mathrm{A}+\mathrm{A}$ collisions from SPS to LHC energies, including electromagnetic probes such as photons and dileptons (Linnyk et al. 2016).

While heavy-ion reactions at RHIC and LHC energies probe a partonic medium at small baryon chemical potential $\mu_{\mathrm{B}}$, the current interest is in collisions at lower bombarding energies (FAIR, NICA) where the net baryon density is higher than $\mu_{\mathrm{B}}$ accordingly. In order to explore the partonic systems at higher $\mu_{\mathrm{B}}$, the PHSD approach is extended to incorporate partonic quasiparticles and their differential cross sections that depend on $T$ and $\mu_{\mathrm{B}}$ explicitly.

\section{2 | THE PHSD APPROACH}

The PHSD transport approach (Bratkovskaya et al. 2011; Cassing \& Bratkovskaya 2009; Linnyk et al. 2013) is an off-shell transport approach based on Kadanoff-Baym equations in the first-order gradient expansion (Cassing 2009) using "resummed" propagators from the dynamical quasiparticle model (DQPM) (Cassing 2007) for the partonic phase. In PHSD, the parton spectral functions $\rho_{j}$ $(j=q, \bar{q}, g)$ are no longer $\delta$-functions in the invariant mass squared as in conventional cascade or transport models but depend on the parton mass and width parameters:

$$
\rho_{j}(\omega, \mathbf{p})=\frac{\gamma_{j}}{E_{j}}\left(\frac{1}{\left(\omega-E_{j}\right)^{2}+\gamma_{j}^{2}}-\frac{1}{\left(\omega+E_{j}\right)^{2}+\gamma_{j}^{2}}\right),
$$

separately for quarks/antiquarks and gluons $(j=q, \bar{q}, g)$. With the convention

$$
E^{2}\left(\mathbf{p}^{2}\right)=\mathbf{p}^{2}+M_{j}^{2}-\gamma_{j}^{2},
$$

the parameters $M_{j}^{2}$ and $\gamma_{j}$ are directly related to the real and imaginary parts of the retarded self-energy, for example, $\Pi_{j}=M_{j}^{2}-2 i \gamma_{j} \omega$.

The actual parameters in Equation (1), that is, the gluon mass $M_{\mathrm{g}}$ and width $\gamma_{\mathrm{g}}$-used as input in the present PHSD calculations-as well as the quark mass $M_{\mathrm{q}}$ and width $\gamma_{\mathrm{q}}$, are depicted in Figure 1 as a function of the scaled temperature $T / T_{\mathrm{c}}$ for different $\mu_{\mathrm{B}}$. These values for the masses and widths have been fixed by fitting the lattice quantum chromodynamics (QCD) results from Borsanyi et al. $(2012,2014)$ in thermodynamic equilibrium.
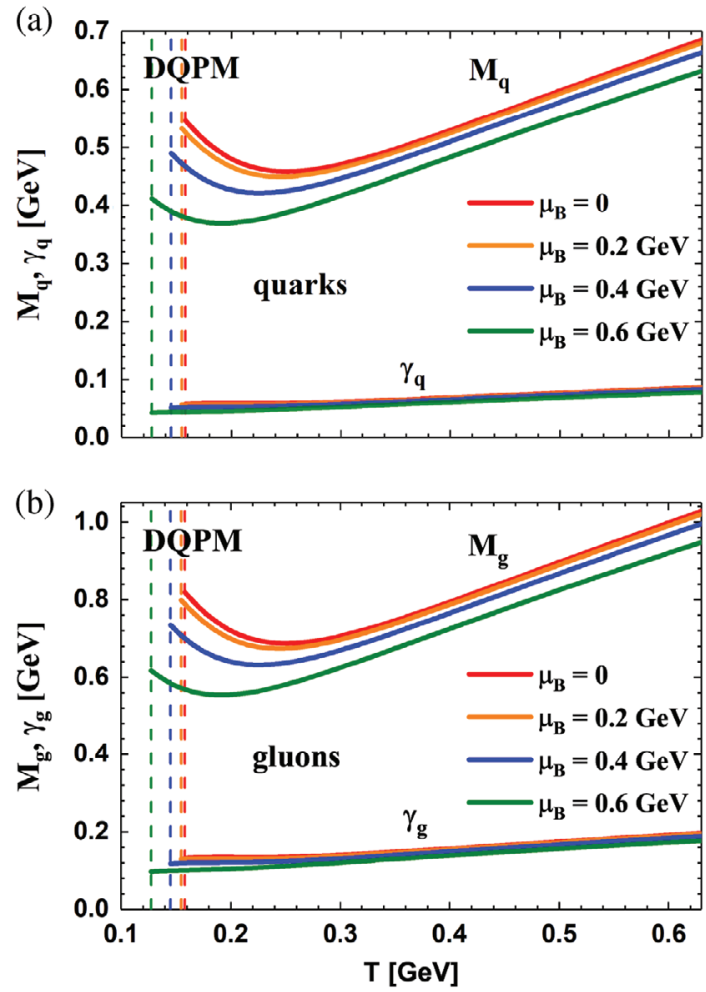

F I G U R E 1 The effective quark (a) and gluon (b) masses $M$ and widths $\gamma$ (from a fit to the entropy density from lattice QCD data) as a function of the temperature $T$ for different $\mu_{B}$. The vertical dashed lines correspond to the dynamical quasiparticle model (DQPM) $\mu_{\mathrm{B}}$-dependent critical temperature $T_{\mathrm{c}}\left(\mu_{\mathrm{B}}\right)$

A scalar mean-field $U_{\mathrm{s}}\left(\rho_{\mathrm{s}}\right)$ for quarks and antiquarks can be defined by the derivative,

$$
U_{\mathrm{s}}\left(\rho_{\mathrm{s}}\right)=\frac{d V_{\mathrm{p}}\left(\rho_{\mathrm{s}}\right)}{d \rho_{\mathrm{s}}},
$$

which is evaluated numerically within the DQPM. Here, $V_{\mathrm{p}}$ is a potential energy density

$$
V_{p}\left(T, \mu_{q}\right)=T_{g_{-}}^{00}\left(T, \mu_{q}\right)+T_{q_{-}}^{00}\left(T, \mu_{q}\right)+T_{\bar{q}-}^{00}\left(T, \mu_{q}\right),
$$

where the different contributions $T_{j-}^{00}$ correspond to the space-like part of the energy-momentum tensor component $T_{j}^{00}$ of parton $j=g, q, \bar{q}$ (cf. Section 3 in Cassing 2007). The scalar mean-field $U_{\mathrm{s}}\left(\rho_{\mathrm{s}}\right)$ for quarks and antiquarks is repulsive as a function of the parton scalar density $\rho_{\mathrm{s}}$ and shows that the scalar mean field is in the order of a few $\mathrm{GeV}$ for $\rho_{\mathrm{s}}>10 \mathrm{fm}^{-3}$. The mean-field (Equation (3)) is used in the PHSD transport calculations and determines the force on a partonic quasiparticle $j$, that is,

$$
\sim M_{j} / E_{j} \nabla U_{\mathrm{s}}(x)=M_{j} / E_{j} d U_{\mathrm{s}} / d \rho_{\mathrm{s}} \nabla \rho_{\mathrm{s}}(x),
$$

where the scalar density $\rho_{\mathrm{s}}(x)$ is determined numerically on a space-time grid. Furthermore, a two-body interaction strength can be extracted from the DQPM and from the quasiparticle width in line with Peshier \& 
Cassing (2005). The transition from partonic to hadronic degrees-of-freedom (and vice versa) is described by covariant transition rates for the fusion of quark-antiquark pairs or three quarks (antiquarks), respectively, obeying flavor current conservation and color neutrality, as well as energy-momentum conservation (Cassing \& Bratkovskaya 2009). As the dynamical quarks and antiquarks become very massive close to the phase transition, the formed resonant "pre-hadronic" color-dipole states ( $q \bar{q}$ or $q q q$ ) are of high invariant mass too and sequentially decay to the ground-state meson and baryon octets, thus increasing the total entropy.

On the hadronic side, PHSD explicitly includes the baryon octet and decouplet, the $0^{-}$- and $1^{-}$-meson nonets, and selected higher resonances as in the Hadron-String-Dynamics (HSD) approach (Bratkovskaya et al. 2004a, 2004b; Cassing 2002; Cassing \& Bratkovskaya 1999). Note that PHSD and HSD (without explicit partonic degrees-of-freedom) merge at low energy density, particularly below the local critical energy density $\varepsilon_{c} \approx$ $0.5 \mathrm{GeV} \mathrm{fm}^{-3}$.

\subsection{Initial conditions}

The initial conditions for the parton/hadron dynamical system have to be specified additionally. In order to describe relativistic heavy-ion reactions, we start with two nuclei in their "semi-classical" ground state, boosted toward each other with a velocity $\beta$ (in $z$-direction), fixed by the bombarding energy. The initial phase-space distributions of the projectile and target nuclei are determined in the local Thomas-Fermi limit as in the HSD transport approach (Cassing \& Bratkovskaya 1999) or the Ultra-relativistic Quantum-Molecular Dynamics model (Bass et al. 1998a, 1998b). We recall that, at relativistic energies, the initial interactions of two nucleons are well described by the excitation of two color-neutral strings that decay in time to the known hadrons (mesons, baryons, antibaryons) (Bengtsson \& Sjostrand 1987). Initial hard processes-that is, the short-range high-momentum transfer reactions that can be well described by perturbative QCD—are treated in PHSD (as in HSD) via PYTHIA. The novel element in PHSD (relative to HSD) is the "string melting concept" as also used in the a multi-phase transport model (Lin et al. 2005) in a similar context. However, in PHSD, the strings (or possibly formed hadrons) are only allowed to 'melt' if the local energy density $\epsilon(x)$ (in the local rest frame) is above the transition energy density $\epsilon_{\mathfrak{c}}$, which in the DQPM is $\epsilon_{\mathrm{c}} \approx 0.5 \mathrm{GeV} \mathrm{fm}^{-3}$. The mesonic strings then decay to quark-antiquark pairs according to an intrinsic quark momentum distribution,

$$
F(\mathbf{q}) \sim \exp \left(-2 b^{2} \mathbf{q}^{2}\right),
$$

in the meson rest frame. The parton final four momenta are selected randomly according to the momentum distribution (Equation (6)) (with $b=0.66 \mathrm{fm}$ ), and the parton-energy distribution is fixed by the DQPM at a given energy density $\epsilon\left(\rho_{\mathrm{s}}\right)$ in the local cell with scalar parton density $\rho_{\mathrm{s}}$. The flavor content of the $q \bar{q}$ pair is fully determined by the flavor content of the initial string. Through construction, the "string melting" to massive partons conserves energy and momentum, as well as the flavor content. In contrast to Lin et al. (2005), the partons are of finite mass-in line with their local spectral function - and additionally obtain a random color $c=(1,2,3)$ or $(r, b, g)$. Of course, the color appointment is color neutral, that is, when selecting a color $c$ for the quark randomly, the color for the antiquark is fixed by $-c$. The baryonic strings melt analogously into a quark and a diquark, while the diquark, furthermore, decays to two quarks. Dressed gluons are generated by the fusion of nearest neighbor $q+\bar{q}$ pairs $(q+\bar{q} \rightarrow g)$ that are flavor neutral until the ratio of gluons to quarks reaches the value $N_{g} /\left(N_{q}+N_{\bar{q}}\right)$ given by the DQPM for the energy density of the local cell. This "recombination" is performed for all cells in space during the passage time of the target and projectile (before the calculation continues with the next time step) and conserves the four-momentum as well as the flavor currents. We note, however, that the initial phase in PHSD is dominated by quark and antiquark degrees of freedom.

\section{2 | Partonic cross sections}

On the partonic side, the following elastic and inelastic interactions are included in PHSD $q q \leftrightarrow q q, \bar{q} \bar{q} \leftrightarrow \bar{q} \bar{q}$, $g g \leftrightarrow g g, g g \leftrightarrow g, q \bar{q} \leftrightarrow g, q g \leftrightarrow q g, g \bar{q} \leftrightarrow g \bar{q}$ exploiting "detailed-balance" with cross sections calculated from the leading Feynman diagrams using the effective propagators and couplings $g^{2}\left(T / T_{c}\right)$ from the DQPM (Moreau et al. 2019).

Partonic reactions such as $g+q \leftrightarrow q$ or $g+g \leftrightarrow q+\bar{q}$ have been discarded in the present calculations due to their low rates as the large mass of the gluon leads to a strong mismatch in the energy thresholds between the initial and final channels. In this case, $q$ stands for the four lightest quarks $(u, d, s, c)$. Furthermore, the evaluation of photon and dilepton production is calculated perturbatively, and channels such as $g+q \rightarrow q+\gamma$ are included. In this case, the probability for photon (dilepton) production from each channel is added up and integrated over space and time (Linnyk et al. 2016) without introducing any new parameter in the PHSD approach as the electromagnetic coupling is well known. 
Numerical tests of the parton dynamics with respect to conservation laws and interaction rates in and out of equilibrium in a finite box with periodic boundary conditions have been presented in Ozvenchuk et al. (2013a). In fact, in Ozvenchuk et al. (2013a), it was shown that the PHSD calculations "in the box" give practically the same results in equilibrium as the DQPM. We note in passing that the total energy is conserved in the box calculations up to about three digits, while in the heavy-ion collisions addressed here, the violation of energy conservation is typically less than 1\% (Cassing \& Bratkovskaya 2009).

\section{I TRANSPORT PROPERTIES OF THE PARTONIC SYSTEM}

The starting point to evaluate viscosity coefficients of partonic matter is the Kubo formalism (Kubo 1957), which was also used to calculate the viscosities within the PHSD in a box with periodic boundary conditions (cf. Ref. [Ozvenchuk et al. 2013b]). We focus here on the calculation of the shear viscosity based on Aarts \& Martinez Resco (2002), which reads:

$$
\begin{aligned}
\eta^{\mathrm{Kubo}}\left(T, \mu_{q}\right)= & -\int \frac{d^{4} p}{(2 \pi)^{4}} p_{x}^{2} p_{y}^{2} \\
& \times \sum_{i=q, \bar{q}, g} d_{i} \frac{\partial f_{i}(\omega)}{\partial \omega} \rho_{i}(\omega, \mathbf{p})^{2},
\end{aligned}
$$

where the notation $f_{i}(\omega)=f_{i}\left(\omega, T, \mu_{\mathrm{q}}\right)$ is used for the distribution functions, and $\rho_{i}$ denotes the spectral function of the partons, while $d_{i}$ stand for the degeneracy factors. We note that the derivative of the distribution function accounts for the Pauli-blocking (-) and Bose-enhancement $(+)$ factors. Following Lang et al. (2012), we can evaluate the integral over $\omega=p_{0}$ in Equation (7) by using the residue theorem. When keeping only the leading order contribution in the width $\gamma\left(T, \mu_{\mathrm{B}}\right)$ from the residue-evaluated at the poles of the spectral function $\omega_{i}= \pm \widetilde{E}(\mathbf{p}) \pm i \gamma$-we finally obtain:

$$
\begin{aligned}
\eta^{\mathrm{RTA}}\left(T, \mu_{q}\right) & =\frac{1}{15 T} \int \frac{d^{3} p}{(2 \pi)^{3}} \\
& \times \sum_{i=q, \bar{q}, g}\left(\frac{\mathbf{p}^{4}}{E_{i}^{2} \Gamma_{i}\left(\mathbf{p}_{i}, T, \mu_{q}\right)} d_{i}\left(\left(1 \pm f_{i}\left(E_{i}\right)\right) f_{i}\left(E_{i}\right)\right)\right),
\end{aligned}
$$

which corresponds to the expression derived in the relaxation time approximation (RTA) (Sasaki \& Redlich 2009) by identifying the interaction rate $\Gamma$ with $2 \gamma$ as expected from transport theory in the quasiparticle limit (Blaizot \& Iancu 1999). We recall that $\gamma$ is the width parameter in

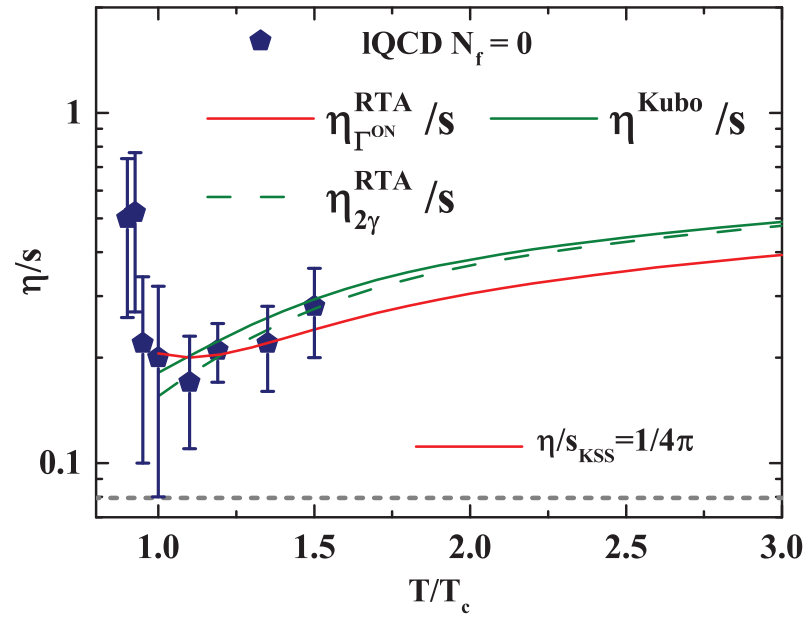

F I G U R E 2 The ratio of shear viscosity to entropy density as a function of the scaled temperature $T / T_{\mathrm{c}}$ for $\mu_{\mathrm{B}}=0$ from Equations (7) and (8). The solid green line ( $\left.\eta^{\text {Kubo }} / s\right)$ shows the results from the original dynamical quasiparticle model (DQPM) in the Kubo formalism, while the dashed green line $\left(\eta_{2 \gamma}^{\mathrm{RTA}} / s\right)$ shows the same result in the relaxation time approximation Equation (8). The solid red line $\left(\eta_{\Gamma^{\text {on }}}^{\mathrm{RTA}} / s\right)$ results from Equation (8) using the interaction rate $\Gamma^{\text {on }}$ calculated by the microscopic differential cross sections in the on-shell limit. The dashed gray line demonstrates the Kovtun-Son-Starinets bound (Kovtun et al. 2005) $(\eta / s)_{\mathrm{KSS}}=1 /(4 \pi)$, and the symbols show IQCD data for pure SU(3) gauge theory taken from Astrakhantsev et al. (2017) (pentagons)

the parton propagator (Equation (1)). The interaction rate $\Gamma_{i}\left(\mathbf{p}_{i}, T, \mu_{q}\right)$ (inverse relaxation time) is calculated microscopically from the collision integral using the differential cross sections for parton scattering as described in Section 2.2. Furthermore, we recall that the pole energy is $E_{i}^{2}=$ $p^{2}+M_{i}^{2}$, where $M_{i}$ is the pole mass given in the DQPM. Here, we use the notation (Tex translation failed), which includes the contribution from all possible partons that, in our case, are the gluons and the (anti-)quarks of three different flavors $(u, d, s)$.

The actual results are displayed in Figure 2 for the ratio of shear viscosity to entropy density $\eta / s$ as a function of the scaled temperature $T / T_{\mathrm{c}}$ for $\mu_{\mathrm{B}}=0$ in comparison to those from lattice QCD (Astrakhantsev et al. 2017). The solid green line $\left(\eta^{\text {Kubo }} / s\right)$ shows the result from the original DQPM in the Kubo formalism, while the dashed green line $\left(\eta_{2 \gamma}^{\mathrm{RTA}} / s\right)$ shows the same result in the RTA (Equation (8)) by replacing $\Gamma_{i}$ by $2 \gamma_{i}$. The solid red line $\left(\eta_{\Gamma \text { on }}^{\mathrm{RTA}} / s\right)$ results from Equation (Equation (8)) using the interaction rate $\Gamma^{\text {on }}$ calculated by the microscopic differential cross sections in the on-shell limit. We find that the ratios $\eta / s$ do not differ very much and have a similar behavior as a function of temperature. The approximation (Equation (8)) of the shear viscosity is found to be very close to the one from the Kubo formalism (Equation (7)), indicating that the quasiparticle limit $(\gamma \ll M)$ holds in the DQPM. 


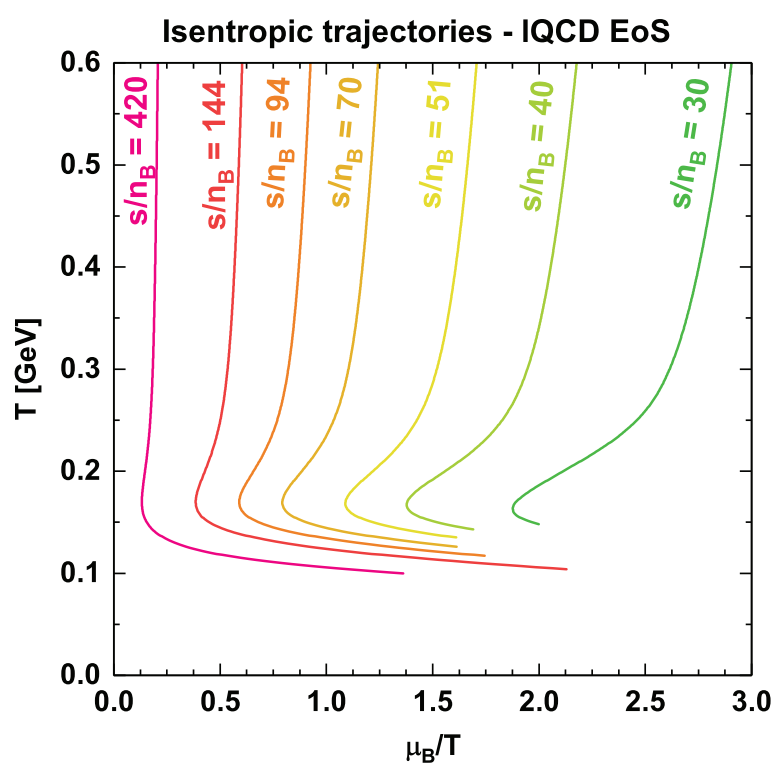

F I G U R E 3 The lines of constant entropy density over baryon density $s / n_{\mathrm{B}}$ in the $\left(T, \mu_{\mathrm{B}} / T\right)$ plane as evaluated by using the lattice EoS

We note in passing that there is no strong variation with $\mu_{\mathrm{B}}$ for fixed $T / T_{\mathrm{c}}\left(\mu_{\mathrm{B}}\right)$; however, the ratio increases slightly with $\mu_{\mathrm{B}}$ in the on-shell limit, while it slightly drops with $\mu_{\mathrm{B}}$ in the Kubo formalism for the DQPM (Moreau et al. 2019). Accordingly, there is some model uncertainty when extracting the shear viscosity in the different approximations.

\section{4 | OBSERVABLES FROM RELATIVISTIC NUCLEUS-NUCLEUS COLLISIONS}

Before coming to the actual PHSD results we show in Figure 3 the lines of constant entropy density over baryon density $s / n_{\mathrm{B}}$ in the $\left(T, \mu_{\mathrm{B}} / T\right)$ plane as evaluated by using the lattice equation-of-state. Here, the high ratio $s / n_{\mathrm{B}}=420$ roughly corresponds to LHC energies, while at FAIR/NICA energies, one has $s / n_{B} \approx 30$. Accordingly, one might find some traces of the explicit $\mu_{\mathrm{B}}$ dependence of the partonic cross sections in observables at FAIR/NICA energies. We mention that, when implementing the differential cross sections and parton masses into the PHSD5.0 approach, one has to specify the" Lagrange parameters" $T$ and $\mu_{\mathrm{B}}$ in each computational cell in space-time. This has been conducted by using the lattice equation of state, which is practically identical to the lattice QCD equation of state, and a diagonalization of the energy-momentum tensor from PHSD as described in Moreau et al. (2019).

Figure 4 displays the actual results for hadronic rapidity distributions in case of $5 \%$ central $\mathrm{Pb}+\mathrm{Pb}$ collisions at $30 \mathrm{~A} \mathrm{GeV}$ for PHSD4.0 (Palmese et al. 2016) (green dot-dashed lines), PHSD5.0 with partonic cross sections and parton masses calculated for $\mu_{\mathrm{B}}=0$ (blue dashed lines), and with cross sections and parton masses evaluated at the actual chemical potential $\mu_{\mathrm{B}}$ in each individual space-time cell (red lines) in comparison to the experimental data from the NA49 Collaboration (Alt et al. 2006, 2008a, 2008b). Here, we focus on the most abundant hadrons, that is, pions, kaons, protons, neutral hyperons, antiprotons, and antihyperons. We note in passing that the effects of chiral symmetry restoration are incorporated as in Palmese et al. (2016) as this was found to be mandatory to achieve a reasonable description of the strangeness degrees of freedom reflected in the kaon and neutral hyperon dynamics. As seen in Figure 4, there is practically no difference in rapidity distributions for all the hadron species from the different versions of PHSD within line width, which implies that there is no sensitivity to the new partonic differential cross sections and parton masses used. A comparison to the available experimental data is included (for orientation) but not discussed explicitly as this has been carried out in more detail in Palmese et al. (2016).

\section{5 | SUMMARY}

In this contribution, we have described the PHSD transport approach (Linnyk et al. 2016) and its recent extension to PHSD5.0 (Moreau et al. 2019) to incorporate differential "off-shell cross sections" for all binary partonic channels that are based on the same effective propagators and couplings as used in the QGP equation of state and the parton propagation. To this end, we have calculated the partonic differential cross sections as a function of $T$ and $\mu_{\mathrm{B}}$ for the leading tree-level diagrams (cf. appendices of Moreau et al. 2019). Furthermore, we have used these differential cross sections to evaluate partonic scattering rates $\Gamma_{i}\left(T, \mu_{\mathrm{B}}\right)$ for fixed $T$ and $\mu_{\mathrm{B}}$, as well as to compute the ratio of the shear viscosity $\eta$ to entropy density $s$ within the Kubo formalism in comparison to calculations from lattice QCD (lQCD). It turns out that the ratio $\eta / s$ calculated with the partonic scattering rates in the RTA is very similar to the original result from the DQPM and to IQCD results such that the present extension of the PHSD approach does not lead to different partonic transport properties. We recall that the novel PHSD version (PHSD5.0) is practically parameter free in the partonic sector as the effective coupling (squared) is determined by a fit to the scaled entropy density from $1 Q C D$. The dynamical masses for quarks and gluons are then fixed by the HTL expressions (Linnyk et al. 2016). The interaction rate in the time-like sector is, furthermore, calculated in leading order using the DQPM propagators and coupling. 
F I G U R E 4 The rapidity distributions for $5 \%$ central $\mathrm{Pb}+\mathrm{Pb}$ collisions at $30 \mathrm{~A} \mathrm{GeV}$ for PHSD4.0 (green dot-dashed lines), PHSD5.0 with partonic cross sections and parton masses calculated for $\mu_{\mathrm{B}}=0$ (blue dashed lines), and with cross sections and parton masses evaluated at the actual chemical potential $\mu_{\mathrm{B}}$ in each individual space-time cell (red lines) in comparison to the experimental data from the NA49 Collaboration (Alt et al. 2006, 2008a, 2008b). All PHSD results are practically the same within the line width

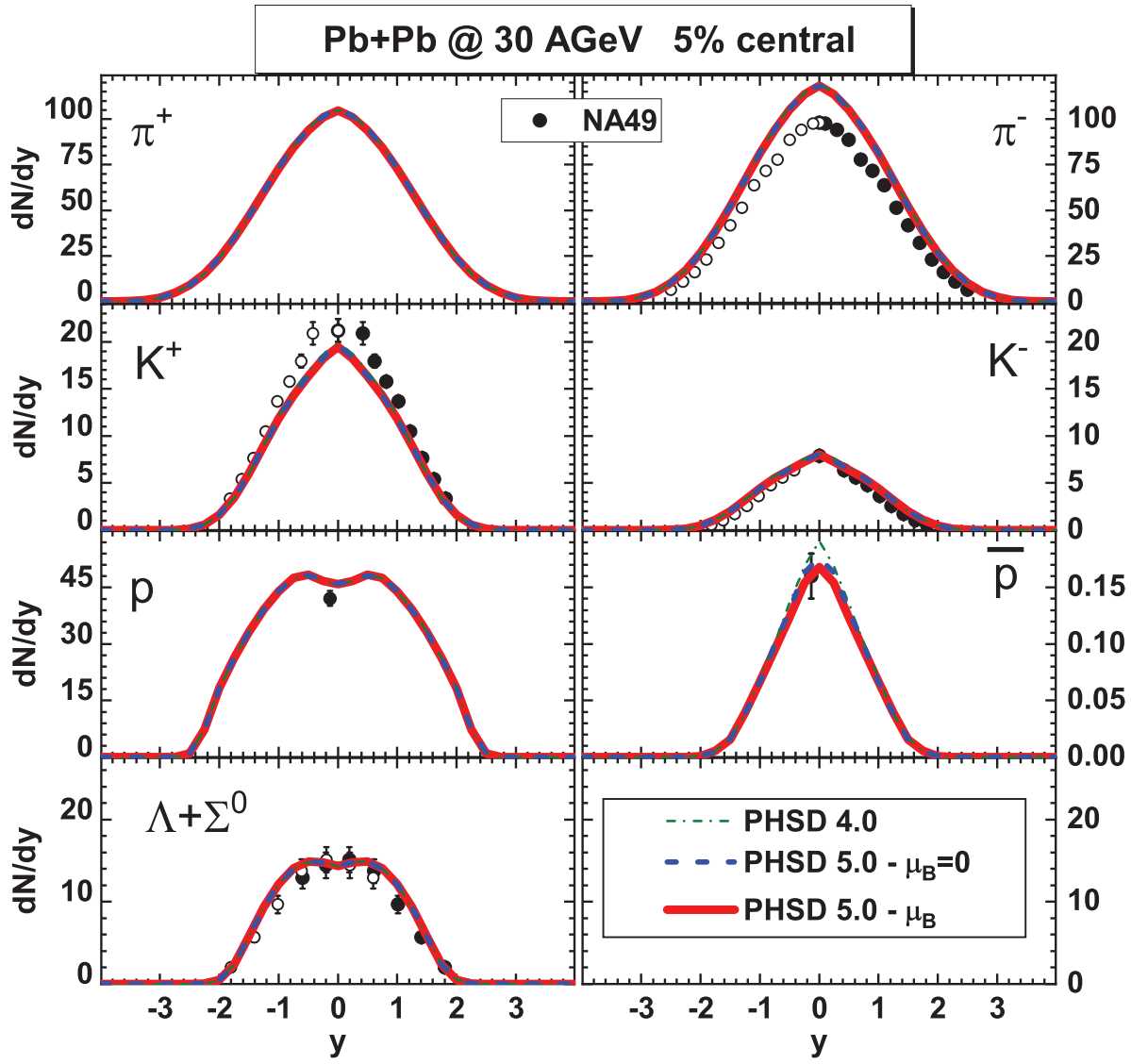

When implementing the differential cross sections and parton masses into the PHSD5.0 approach, one has to specify the "Lagrange parameters" $T$ and $\mu_{\mathrm{B}}$ in each computational cell in space-time. This has been performed by using the lattice equation of state and a diagonalization of the energy-momentum tensor from PHSD as described in Moreau et al. (2019). As an example, we have shown the results for hadronic rapidity distributions from the previous PHSD4.0 (Palmese et al. 2016) with the novel version PHSD5.0 (with and without the explicit dependence of the partonic differential cross sections and parton masses on $\left.\mu_{\mathrm{B}}\right)$ for $5 \%$ central $\mathrm{Pb}+\mathrm{Pb}$ collisions at $30 \mathrm{~A} \mathrm{GeV}$. No differences for all the hadron "bulk" observables from the various PHSD versions have been found at FAIR/NICA energies within line width, which implies that there is no sensitivity to the $\mu_{\mathrm{B}}$-dependence of the new partonic differential cross sections used.

Our findings can be understood as follows: The fact that we find only small traces of the $\mu_{\mathrm{B}}$ dependence of partonic scattering dynamics in heavy-ion "bulk" observables-although the differential cross sections and parton masses clearly depend on $\mu_{\mathrm{B}}$-means that one needs a sizable partonic density and large space-time QGP volume to explore the dynamics in the QGP phase. These conditions are only fulfilled at high bombarding energies (top SPS, RHIC energies) where, however, $\mu_{\mathrm{B}}$ is rather low.
On the other hand, decreasing the bombarding energy to FAIR/NICA energies and, thus, increasing $\mu_{\mathrm{B}}$, leads to collisions that are dominated by the hadronic phase where the extraction of information about the parton dynamics will be rather complicated based on "bulk" observables. Further investigations of other observables (such as flow coefficients $v_{\mathrm{n}}$ of particles and antiparticles, fluctuations, and correlations) might contain more visible " $\mu_{\mathrm{B}}$ traces" from the QGP phase.

\section{ACKNOWLEDGMENTS}

The authors acknowledge inspiring discussions with J. Aichelin, H. Berrehrah, C. Ratti, and T. Steinert. This work was supported by the LOEWE center "HIC for FAIR". Furthermore, P.M., L.O., and E.B. acknowledge support by the Deutsche Forschungsgemeinschaft (DFG, German Research Foundation) through the grant CRC-TR 211 "Strong-interaction matter under extreme conditions"-Project number 315477589-TRR 211. O.S. acknowledges support from HGS-HIRe for FAIR, and L.O. and E.B. thank the COST Action THOR, CA15213. The computational resources have been provided by the LOEWE-Center for Scientific Computing. Open access funding enabled and organized by Projekt DEAL.

\section{REFERENCES}

Aarts, G., \& Martinez Resco, J. M. 2002, JHEP, 04, 053. 
Alt, C., et al. 2006, Phys. Rev., C73, 044910.

Alt, C., et al. 2008a, Phys. Rev., C78, 034918.

Alt, C., et al. 2008b, Phys. Rev., C77, 024903.

Astrakhantsev, N., Braguta, V., \& Kotov, A. 2017, JHEP, 04, 101.

Bass, S. A., et al. 1998a, Progr. Part. Nucl. Phys., 41, 255.

Bass, S. A., et al. 1998b, Progr. Part. Nucl. Phys., 41, 225.

Bengtsson, H.-U., \& Sjostrand, T. 1987, Comput. Phys. Commun., 46, 43.

Blaizot, J.-P., \& Iancu, E. 1999, Nucl. Phys., B557, 183.

Borsanyi, S., Endrodi, G., Fodor, Z., Katz, S. D., Krieg, S., Ratti, C., \& Szabo, K. K. 2012, JHEP, 08, 053.

Borsanyi, S., Fodor, Z., Hoelbling, C., Katz, S. D., Krieg, S., \& Szabo, K. K. 2014, Phys. Lett., B730, 99.

Bratkovskaya, E. L., Bleicher, M., Reiter, M., et al. 2004a, Phys. Rev., C69, 054907.

Bratkovskaya, E. L., Soff, S., Stoecker, H., van Leeuwen, M., \& Cassing, W. 2004b, Phys. Rev. Lett., 92, 032302.

Bratkovskaya, E. L., Cassing, W., Konchakovski, V. P., \& Linnyk, O. 2011, Nucl. Phys., A856, 162.

Cassing, W. 2002, Nucl. Phys., A700, 618.

Cassing, W. 2007, Nucl. Phys., A795, 70.

Cassing, W. 2009, Eur. Phys. J. ST, 168, 3.

Cassing, W., \& Bratkovskaya, E. L. 1999, Phys. Rep., 308, 65.

Cassing, W., \& Bratkovskaya, E. L. 2009, Nucl. Phys., A831, 215.

Kovtun, P., Son, D. T., \& Starinets, A. O. 2005, Phys. Rev. Lett., 94, 111601.

Kubo, R. 1957, J. Phys. Soc. Jpn., 12, 570.

Lang, R., Kaiser, N., \& Weise, W. 2012, Eur. Phys. J., A48, 109.

Lin, Z.-W., Ko, C. M., Li, B.-A., Zhang, B., \& Pal, S. 2005, Phys. Rev., C72, 064901.

Linnyk, O., Konchakovski, V. P., Cassing, W., \& Bratkovskaya, E. L. 2013, Phys. Rev., C88, 034904.

Linnyk, O., Bratkovskaya, E. L., \& Cassing, W. 2016, Progr. Part. Nucl. Phys., 87, 50.
Moreau, P., Soloveva, O., Oliva, L., Song, T., Cassing, W., \& Bratkovskaya, E. 2019, Phys. Rev. C, 100, 014911.

Ozvenchuk, V., Linnyk, O., Gorenstein, M. I., Bratkovskaya, E. L., \& Cassing, W. 2013a, Phys. Rev., C87(2), 024901.

Ozvenchuk, V., Linnyk, O., Gorenstein, M. I., Bratkovskaya, E. L., \& Cassing, W. 2013b, Phys. Rev., C87(6), 064903.

Palmese, A., Cassing, W., Seifert, E., Steinert, T., Moreau, P., \& Bratkovskaya, E. L. 2016, Phys. Rev., C94(4), 044912.

Peshier, A., \& Cassing, W. 2005, Phys. Rev. Lett., 94, 172301.

Sasaki, C., \& Redlich, K. 2009, Phys. Rev., C79, 055207.

\section{AUTHOR BIOGRAPHY}

Elena Bratkovskaya is a Senior researcher at the Theory Group of the GSI Helmholtzzentrum fuer Schwerionenforschung, Darmstadt, Germany and Professor at the University of Frankfurt, Germany. Her scientific interests are related to heavy-ion physics, dynamical transport theory for the description of hadrons and partons in and out of equilibrium, phase transition from hadronic to partonic matter, and properties of quark-gluon plasma.

How to cite this article: Bratkovskaya $\mathrm{E}$, Cassing W, Moreau P, Oliva L, Soloveva O, Song T. Exploring the partonic phase at finite chemical potential within an extended off-shell transport approach. Astron. Nachr. 2019;340:885-891. https://doi.org/ 10.1002/asna.201913708 